\title{
Erratum: Acute kidney injury and acute-on- chronic liver failure classifications in prognosis assessment of patients with acute decompensation of cirrhosis
}

Angeli P, Rodríguez E, Piano S, et al. Acute kidney injury and acute-on-chronic liver failure classifications in prognosis assessment of patients with acute decompensation of cirrhosis. Gut 2015;64:1616-22. The funding statement has been updated to read: Part of the work reported in this study has been funded by the project PI12/00330, integrated in the Plan Nacional I $+\mathrm{D}+\mathrm{I}$ and cofunded by ISCIII-Subdirección General de Evaluación and European Regional Development Fund (ERDF).

Gut 2016;65:1394. doi:10.1136/gutjnl-2014-307526corr1 